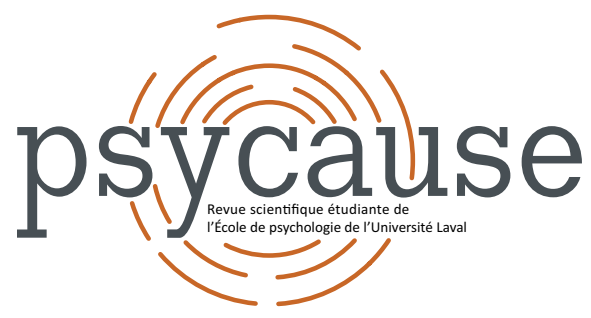

\title{
PSYCAUSE
}

Revue scientifique étudiante de l'École de psychologie de l'Université Laval

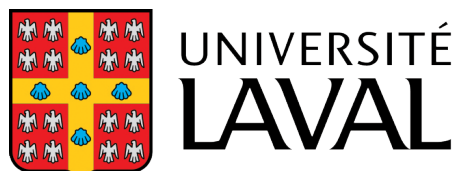

Faculté des sciences sociales École de psychologie

NOVEMBRE 2020 - VOL. $10 \mathrm{~N}^{\circ} 2$

\section{LE RECRUTEMENT DANS LA RECHERCHE SUR LE JEU AVEC DES MISES D’ARGENT PERSONNEL}

Camille CÔTÉ-THIBEAULT ${ }^{1}$, Catherine LAMIRANDE ${ }^{1}$, Coralie BEAULIEU ${ }^{1}$, Benjamin GALIPEAU ${ }^{1}$, Christian JACQUES ${ }^{1} \&$ Isabelle GIROUX

1 École de psychologie, Université Laval

*camille.cote-thibeault.1@ulaval.ca

\section{Pour citer l'article}

Côté-Thibeault, C., Lamirande, C., Beaulieu, C., Galipeau, B., Jacques, C., \& Giroux, I. (2020). Le recrutement dans la recherche sur le jeu avec des mises d'argent personnel. Psycause: Revue scientifique étudiante de l'École de psychologie de l'Université Laval, 10(2), 19-20. 


\title{
LE RECRUTEMENT DANS LA RECHERCHE SUR LE JEU AVEC DES MISES D'ARGENT PERSONNEL
}

\author{
Camille CÔTÉ-THIBEAULT ${ }^{1}$, Catherine LAMIRANDE¹, Coralie BEAULIEU ${ }^{1}$, Benjamin GALIPEAU ${ }^{1}$, Christian JACQUES ${ }^{1}$ \& \\ Isabelle GIROUX' \\ ${ }^{1}$ École de psychologie, Université Laval \\ * camille.cote-thibeault.1@ulaval.ca
}

\section{Mots clés : Jeu de hasard et d'argent, méthodologie, mises d'argent personnel, recrutement}

Les mesures de jeu responsable servent à limiter et prévenir les conséquences négatives associées aux jeux de hasard et d'argent (JHA; Blaszczynski, Ladouceur, \& Shaffer, 2004). Pour s'assurer que ces mesures soient efficaces, il est essentiel de les tester avant leur implantation. Le défi des chercheurs, qui réalisent généralement ces études en laboratoire, est d'améliorer la validité externe, afin d'optimiser l'efficacité des mesures préventives une fois implantées dans les milieux de jeu. Une compensation financière garantie est couramment offerte aux participants dans un contexte d'étude, mais ce montant d'argent diminuerait le risque perçu par le joueur (Anderson \& Brown, 1984). Cependant, dans un contexte réel de jeu, il n'y a aucune garantie de gain. Ainsi, certains auteurs proposent de répliquer leur étude dans un contexte où les participants pourraient miser leur argent personnel, en ayant autant la possibilité de gagner de l'argent que d'en perdre (Jardin \& Wulfert, 2012; Montes \& Weatherly, 2017). Afin de déterminer si ce changement méthodologique représenterait un obstacle au recrutement compte tenu du risque encouru, le premier objectif de cette étude est de sonder l'intérêt des joueurs à miser leur propre argent dans un contexte de jeu en laboratoire. Le deuxième objectif est de déterminer si une compensation financière garantie changerait la décision des participants qui refuseraient de miser leur argent personnel. Le troisième objectif est d'identifier des caractéristiques qui prédiront l'acceptation ou le refus de miser son argent personnel.

\section{Méthode}

Cent soixante-quinze participants recrutés par le biais d'annonces dans un journal et d'affiches ont répondu à quatre questionnaires en ligne sur LimeSurvey ou par téléphone. Parmi ceux-ci, 118 d'entre eux jouaient aux appareils de loterie vidéo (ALV), 29 au poker en ligne, 44 au poker sur table et 69 aux jeux de table. Les participants devaient répondre aux questionnaires suivants: (1) Questionnaire sociodémographique ; (2) Gambling Participation Instrument - adapté (Williams, Volberg, Stevens, Williams, \& Arthur, 2017); (3) L'Indice canadien du jeu excessif (Ferris \& Wynne, 2001) et (4) Scénarios. Le questionnaire Scénarios, construit afin de déterminer combien de participants seraient prêts à miser leur argent personnel dans une étude sur les JHA, propose des scénarios d'études impliquant la mise d'argent personnel en laboratoire, l'observation en milieu de jeu réel et l'utilisation de crédits virtuels en laboratoire, selon le type de jeu. Ce questionnaire évalue l'effet d'une compensation financière garantie sur la décision. Les raisons des refus des scénarios proposés sont également examinées. Comme compensation, les participants courraient la chance de gagner un chèque cadeau de $50 \$$ échangeable parmi une sélection de commerces.

\section{Résultats et discussion}

Quatre-vingt-six participants sur 174 , soit $49,1 \%$ de l'échantillon, acceptaient de parier leur argent personnel pour au moins un des types de jeux proposés, dans un projet de recherche portant sur les JHA. Lorsqu'une compensation financière garantie était offerte, 39 participants ayant initialement refusé de parier leur argent personnel changeaient d'avis. Le Tableau 1 montre des résultats supplémentaires du questionnaire Scénarios selon le type de jeu des participants.

La Figure 1 présente, pour chaque type de jeu, le pourcentage d'acceptation de miser son argent personnel en fonction de la compensation financière garantie souhaitée. En général, plus la compensation financière garantie augmente, plus le pourcentage d'acception est grand, et ce, peu importe le type de jeu. En offrant une compensation garantie, presque la moitié des participants ayant initialement refusé changeaient d'avis.

Onze prédicteurs portant sur les comportements de jeu, les caractéristiques sociodémographiques et les types de jeux des participants ont été sélectionnés et analysés par le biais d'un modèle de régression logistique pour déterminer lesquels permettraient de prédire l'acceptation ou le refus de miser son argent personnel (variable dépendante). II en ressort que jouer au poker sur table et miser des montants mensuels élevés dans le jeu permettaient de prédire l'acceptation de miser son argent personnel dans une étude en laboratoire sur les JHA. Jouer au poker serait associé à certains traits de personnalité comme le narcissisme, la compétitivité et la recherche de sensations qui pourraient rendre ceux pratiquant ce jeu plus enclins à participer à une 
Tableau 1

Décision de participer à l'étude selon le scénario proposé et le type de jeu

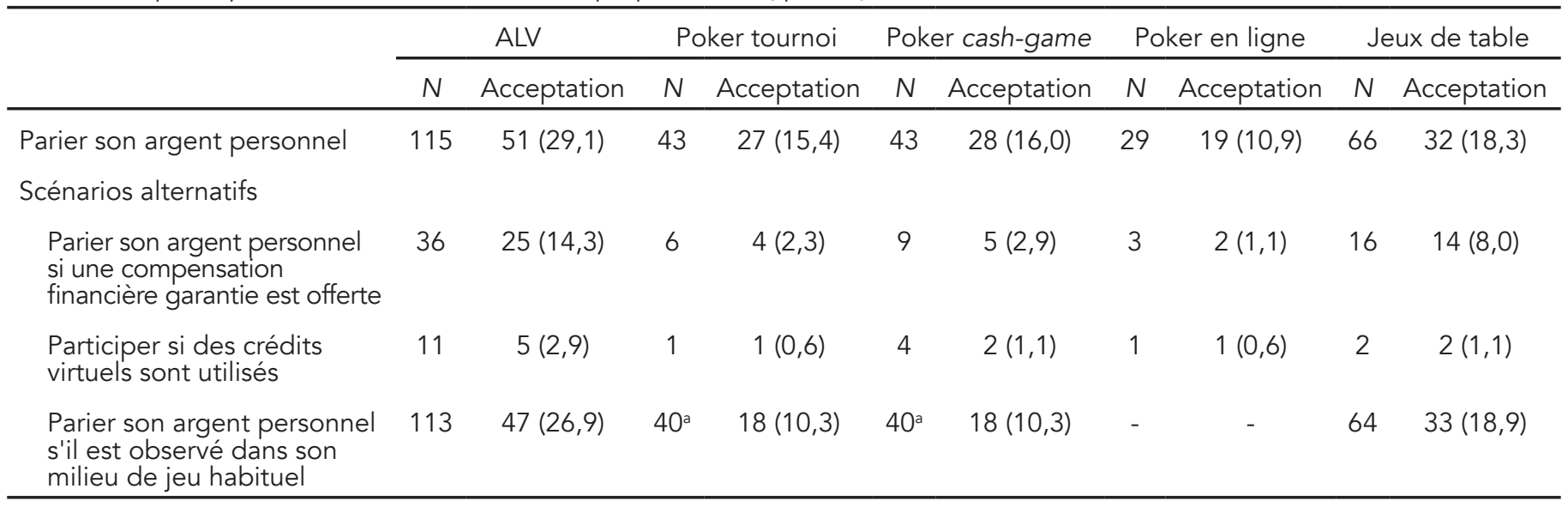

Note. Un même participant peut avoir répondu à plusieurs scénarios. Les valeurs entre parenthèses représentent le pourcentage par rapport à l'ensemble des participants recrutés.

${ }^{a}$ Ce scénario a été proposé à tous les participants ayant joué au poker sur table, sans égard au format tournoi ou au format cash-game.

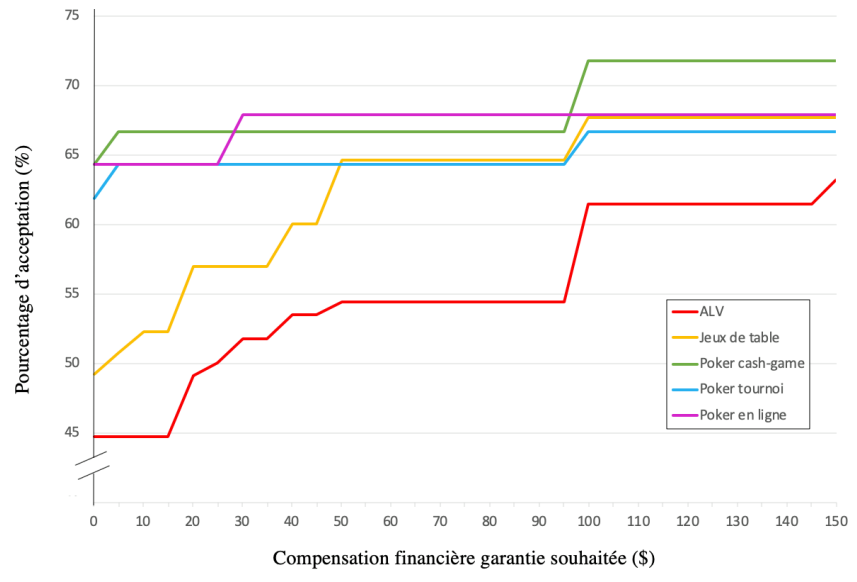

Figure 1. Pourcentage d'acceptation de miser son argent personnel dans une recherche sur le jeu selon la compensation financière garantie est offerte, en fonction du type de jeu joué, soit les ALV $(N=76)$, les jeux de table $(N=46)$, le poker sur table sous forme de cash-game $(N=33)$ ou de tournoi $(N=$ 31) et le poker en ligne $(N=21)$.

telle étude (Lévesque, 2017). Les joueurs qui ont des mises mensuelles élevées sont plus impliqués dans le jeu et seraient donc portés à miser davantage leur argent personnel. Ce sont à ces joueurs que les mesures de prévention s'avéreraient utiles, ce qui signifie que les mesures de jeu responsable pourraient être adaptées à la population pouvant en bénéficier le plus.

\section{Références}

Anderson, G., \& Brown, R. I. F. (1984). Real and laboratory gambling, sensation-seeking and arousal. British Journal of Psychology, 75(3), 401-410. https://doi. org/10.1111/j.2044-8295.1984.tb01910.x
Blaszczynski, A., Ladouceur, R., \& Shaffer, H. J. (2004). A science-based framework for responsible gambling: The Reno Model. Journal of Gambling Studies, 20(3), 301-317. https://doi.org/1050-5350/04/0900-0301

Ferris, J., \& Wynne, H. (2001). L'indice canadien du jeu excessif: Rapport final. Repéré à http://www.jogoremoto.pt/docs/ extra/Jbsm2N.pdf

Jardin, B. F., \& Wulfert, E. (2012). The use of messages in altering risky gambling behavior in experienced gamblers. Psychology of Addictive Behaviors, 26(1), 166-170. https://doi. org/10.1037/a0026202

Lévesque, D. (2017). Personnalité, symptômes anxio-dépressifs et distorsions cognitives: Comparaison de joueurs de poker et de joueurs d'appareils de loterie vidéo. (Thèse de doctorat, Université Laval, Québec, Québec). Repéré à https://corpus.ulaval.ca/jspui/bitstr eam/20.500.11794/28015/1/33182.pdf

Montes, K.S., \&Weatherly, J. N.(2017). Differencesinthegambling behavior of online and non-online student gamblers in a controlled laboratory environment. Journal of Gambling Studies, 33(1), 85-97. https://doi.org/10.1007/ s10899-016-9613-y

Williams, R. J., Volberg, R. A., Stevens, R. M. G., Williams, L. A., \& Arthur, J. N. (2017). The definition, dimensionalization and assessment of gambling participation. Report prepared for the Canadian Consortium for Gambling Research. February 1, 2017. Repéré à https://opus.uleth.ca/bitstream/ handle/10133/4838/Williams\%20the \%20defn,\%20dimensionalization \%20and \%20assessment.pdf

\section{Pour citer l'article}

Côté-Thibeault, C., Lamirande, C., Beaulieu, C., Galipeau, B., Jacques, C., \& Giroux, I. (2020). Le recrutement dans la recherche sur le jeu avec des mises d'argent personnel. Psycause: Revue scientifique étudiante de l'École de psychologie de I'Université Laval, 10(2), 19-20. 